\title{
Structure and mode of action of cyclic lipopeptide pseudofactin II with divalent metal ions
}

\author{
Tomasz Janek $^{\mathrm{a}, *}$, Lígia R. Rodrigues ${ }^{\mathrm{b}}$, Eduardo J. Gudiña ${ }^{\mathrm{b}}$, Żaneta Czyżnikowska $^{\mathrm{a}}$ \\ a Department of Inorganic Chemistry, Faculty of Pharmacy, Wroclaw Medical University, Borowska 211a, 50-556 Wroclaw, Poland \\ ${ }^{\mathrm{b}}$ Centre of Biological Engineering, University of Minho, Campus de Gualtar, 4710-057 Braga, Portugal
}

\section{A R T I C L E I N F O}

\section{Article history:}

Received 12 January 2016

Received in revised form 22 June 2016

Accepted 27 June 2016

Available online 28 June 2016

\section{Keywords:}

Lipopeptides

Pseudofactin II

Metal-ion interaction

Circular dichroism

Molecular modelling

Uropathogenic microorganisms

\begin{abstract}
A B S T R A C T
The interaction of natural lipopeptide pseudofactin II with a series of doubly charged metal cations was examined by matrix-assisted laser-desorption ionization-time of flight (MALDI-TOF) mass spectrometry and molecular modelling. The molecular modelling for metal-pseudofactin II provides information on the metal-peptide binding sites. Overall, $\mathrm{Mg}^{2+}, \mathrm{Ca}^{2+}$ and $\mathrm{Zn}^{2+}$ favor the association with oxygen atoms spanning the peptide backbone, whereas $\mathrm{Cu}^{2+}$ is coordinated by three nitrogens. Circular dichroism (CD) results confirmed that $\mathrm{Zn}^{2+}$ and $\mathrm{Cu}^{2+}$ can disrupt the secondary structure of pseudofactin II at high concentrations, while $\mathrm{Ca}^{2+}$ and $\mathrm{Mg}^{2+}$ did not essentially affect the structure of the lipopeptide. Interestingly, our results showed that the addition of $\mathrm{Zn}^{2+}$ and $\mathrm{Cu}^{2+}$ helped smaller micelles to form larger micellar aggregates. Since pseudofactin II binds metals, we tested whether this phenomena was somehow related to its antimicrobial activity against Staphylococcus epidermidis and Proteus mirabilis. We found that the antimicrobial effect of pseudofactin II was increased by supplementation of culture media with all tested divalent metal ions. Finally, by using Gram-positive and Gram-negative bacteria we showed that the higher antimicrobial activity of metal complexes of pseudofactin II is attributed to the disruption of the cytoplasmic membrane.
\end{abstract}

(C) 2016 Elsevier B.V. All rights reserved.

\section{Introduction}

Lipopeptides constitute a structurally diverse group of metabolites produced by various bacterial and fungal genera via non-ribosomal pathways [1,2]. Structurally they are amphiphilic molecules that comprise both hydrophobic and hydrophilic moieties, being the apolar component usually an alkyl chain, whereas the polar part, more variable, can be ionic (anionic or cationic) or non-ionic. In the past decades, research on lipopeptides has been focused on their antimicrobial, antitumor and surfactant activities.

Pseudofactin II is a cyclic lipooctapeptide (Gly-Ser-Thr-Leu-LeuSer-Leu-Leu) with a palmitic acid connected to the terminal amino group of the eighth amino acid in the peptide moiety. The Cterminal carboxylic group of the last amino acid forms a lactone with the hydroxyl of Thr3 [3]. One of the most advantageous property that has been reported for pseudofactin II is its ability to inhibit the bacterial adhesion (the first stage of biofilm formation) of Escherichia coli, Enterococcus faecalis, Enterococcus hirae, Staphylococcus epidermidis, Proteus mirabilis, as well as the adhesion of

\footnotetext{
* Corresponding author.

E-mail address: tomasz.janek@umed.wroc.pl (T. Janek).
}

Candida albicans to a plate surface [4]. On the other hand, as we have previously reported, melanoma A375 cells are sensitive to the cytotoxic effects of pseudofactin II. In contrast to cancer cells, normal human dermal fibroblast cells (NHDF) showed a lower sensitivity to the antiproliferative effects of this lipopeptide [5].

Metal ions play a vital role in many biological processes, in particular the divalent metallic cations magnesium, calcium, zinc and copper. The interactions of metal ions with favored binding sites (i.e. with specific amino acid residues or sequence motifs) are also known to induce relevant structural changes, for example enzymatic activity is regulated by metal binding [6]. Metal ions, such as $\mathrm{Mg}^{2+}$ in nucleotide complexes [7], have an important structural function whereas $\mathrm{Zn}^{2+}$ participates in the catalytic mechanism of many enzymes, e.g. aminopeptidase [8]. The $\mathrm{Cu}^{2+}$ ion is the most familiar redox metal aiding biological functions [9], while $\mathrm{Ca}^{2+}$ interacts with several proteins playing an important role as secondary messenger in cells [10].

The chemistry of metal coordination compounds is nowadays more popular than before, particularly in the design of more biologically active drugs. Several metal chelates are known to possess antibacterial, antifungal, antiviral and anticancer activity [11-13]. Indeed, in several cases, the metal chelates have been found to be more antimicrobial than the chelating agents themselves. Naturally 
occurring cyclic peptides and lipopeptides such as colistin [14-16], polymyxin B [17] and daptomycin [18,19] are known to bind ions and mediate their transport across natural and prepared biological membranes.

The conformational changes that facilitate ion binding and transport can be studied in considerable detail using molecular dynamics calculations [20], circular dichroism (CD) [21] and Xray crystallographic techniques [22], and such studies provide important insights on the mechanisms underlying ion binding and transport through membranes at the molecular level. Additionally, mass spectrometry (MS) techniques can also unravel metal binding interactions.

As far as we know, this is the first report on the molecular interaction between divalent metal ions and pseudofactin II, as evaluated by MALDI-TOF MS. The effect of metal ions on the secondary structure of the amphipathic lipopeptide was examined by CD spectroscopy. Moreover, experimental data were supported with the results of semi-empirical calculations to determine the possible structures of formed $\mathrm{Me}^{2+}$-pseudofactin systems.

In this work, the interaction between the pseudofactin II and metal ions was studied by surface tension and dynamic light scattering (DLS) measurements. Such a study provided some ideas for the further lipopeptide purification and its potential use in medical applications. In addition, to understand the role of divalent cations in the pseudofactin II activity, as well as to determine how it interacts with bacterial cells membranes, structural studies were essential. The hypothesis of this study was that the metal-peptide complexes formed between the $\mathrm{Mg}^{2+}, \mathrm{Ca}^{2+}, \mathrm{Zn}^{2+}$ or $\mathrm{Cu}^{2+}$ and pseudofactin II bind to cell surfaces and subsequently disrupt the integrity of the cytoplasmic membrane, thus leading to cell death. To prove this hypothesis, we tested the pseudofactin II complexes against multidrug-resistant microorganisms.

\section{Material and methods}

\subsection{Isolation and purification of pseudofactin II}

The Pseudomonas fluorescens BD5 [3] was a kind gift of Dr. Marcin Łukaszewicz from the culture collection of Laboratory of Biotransformation, University of Wroclaw. Strain BD5 was grown on mineral salts medium (MSM; $7 \mathrm{~g} \mathrm{~L}^{-1} \mathrm{~K}_{2} \mathrm{HPO}_{4}, 2 \mathrm{~g} \mathrm{~L}^{-1} \mathrm{KH}_{2} \mathrm{PO}_{4}$, $1 \mathrm{~g} \mathrm{~L}^{-1}\left(\mathrm{NH}_{4}\right)_{2} \mathrm{SO}_{4}, 0.5 \mathrm{~g} \mathrm{~L}^{-1}$ sodium citrate $2 \mathrm{H}_{2} \mathrm{O}$, and $0.1 \mathrm{~g} \mathrm{~L}^{-1}$ $\left.\mathrm{MgSO}_{4} \cdot 7 \mathrm{H}_{2} \mathrm{O}\right)$ supplemented with $2 \%(\mathrm{w} / \mathrm{v})$ D-glucose at $28^{\circ} \mathrm{C}$ for $120 \mathrm{~h}$. Subsequently, the cells were removed by centrifugation (4700g, $10 \mathrm{~min}$ ) and $500 \mathrm{~mL}$ of the cell-free supernatant were used to extract the lipopeptide pseudofactin II with two volumes of ethyl acetate.

The extract was evaporated under vacuum and the crude lipopeptide was subjected to reversed-phase high-performance liquid chromatography (RP-HPLC) as reported earlier [3]. The purified pseudofactin II fraction was dried and stored at $-20^{\circ} \mathrm{C}$ for further studies. Mass spectrometry (data not shown) of the purified pseudofactin II revealed over $99 \%$ purity.

\subsection{Mass spectrometry}

MALDI-TOF mass spectrometry experiments were performed in positive ion mode on an MDS SCIEX 4800 MALDI-TOF/TOF instrument (Applied Biosystem, Foster, CA, USA). Lyophilized HPLC purified pseudofactin II was dissolved in aqueous solution, and the $\mathrm{pH}$ was buffered with ammonium acetate $(\mathrm{pH}=7.4)$. The purified lipopeptide ( $1 \mathrm{mM}$ ) was incubated with $\mathrm{CaCl}_{2}, \mathrm{MgSO}_{4}, \mathrm{CuSO}_{4}$ and $\mathrm{ZnSO}_{4}$. In this competition assay, all the four divalent metal ions (molar ratio peptide: metal $=1: 1$ and $1: 2$ ) were used. The samples were mixed with an equal volume of matrix solution, i.e.
0.1\% a-cyano-4-hydroxycinnamic acid in acetonitrile-water-TFA (50:50:0.01, v/v/v), and left to dry at room temperature. Masses in the range 600-4000 Da were measured.

\subsection{UV-vis absorption spectroscopy}

Absorption spectra of $\mathrm{Cu}^{2+}$-complexes were recorded on a Varian Carry 50 Bio spectrophotometer (Varian, USA) between 200 and $800 \mathrm{~nm}$ using quartz cuvettes ( $1 \mathrm{~cm}$ light path).

\subsection{Circular dichroism spectroscopy}

CD spectra were recorded on a Jasco model J-1500 spectropolarimeter (JASCO, Tokyo, Japan) at $25^{\circ} \mathrm{C}$ under a constant flow of nitrogen gas. Typically, a cell with a $0.5 \mathrm{~cm}$ path length was used for spectra recorded between 190 and $500 \mathrm{~nm}$ with sampling points every $2 \mathrm{~nm}$. The spectra represent the average of 6-9 scans. $\mathrm{CD}$ intensities are expressed in $\Delta \varepsilon\left(\mathrm{dm}^{3} \mathrm{M}^{-1} \mathrm{~cm}^{-1}\right)$. The analysis of secondary structural contents was performed using K2D3 web server. K2D3 web server is an online tool used to assess the secondary structural elements in the form of $\alpha$-helix and $\beta$-strand from the far-UV CD spectra ranging from 190 to $240 \mathrm{~nm}$ [23].

\subsection{Molecular modelling}

To propose the possible structure of the complexes studied we examined ten different conformers of the pseudofactin complexes with the divalent cations $\mathrm{Cu}^{2+}, \mathrm{Zn}^{2+}, \mathrm{Mg}^{2+}$ and $\mathrm{Ca}^{2+}$. Geometries of all complexes have been fully optimized using the NDDO-type semi-empirical method referred to as PM6 which offers a reasonable compromise between accuracy and computational effort [24]. As shown by Stewart, in the case of many properties (including equilibrium geometries, electric-dipol properties and heats of formation) the PM6 method improves substantially upon PM3 method. This method is also very useful to determine structures of bioinorganic complexes with transition metals and modelling of proteins and metaloproteins [24]. Geometry optimizations have been followed by the evaluation of the hessian matrix to confirm that the obtained structures correspond to minima on the potential energy surface. To account for solvent effects (water solution) we applied the polarizable continuum model (PCM) [25-27]. The lowest energy conformers obtained using the PM6 method are described in the present study. All calculations have been performed within unrestricted formalism using Gaussian 09 suite of programs [28].

\subsection{Micelles size measurement by dynamic light scattering}

The mean particle size and polydispersity index (PDI) of micelles formed under different concentrations of metal ions with the lipopeptide diluted in Milli-Q water were obtained by DLS using a Malvern Zetasizer Nano-ZS (Malvern Instruments Ltd., Worcestershire, UK) at $25^{\circ} \mathrm{C}$. The $\mathrm{pH}$ of the solutions was $\sim 6.8-7.0$. The refractive index of the sample was found to be 1.330 . The scattering angle was $173^{\circ}$. The polydispersity index qualifies the particle size distribution, which here ranged from 0 for monodispersed to 1.0 for entirely heterodispersed emulsions. Each sample was analyzed nine times and information about size distribution by intensity, number and volume was recorded.

\subsection{Surface tension measurement}

Lipopeptide and metal ions were dissolved in demineralized water and mixed to obtain several mixtures containing a constant lipopeptide concentration (equal to its critical micelle concentration (CMC), $0.07 \mathrm{mM}$ ) but different metal ions concentrations. The 
concentration of $\mathrm{Mg}^{2+}, \mathrm{Ca}^{2+}, \mathrm{Zn}^{2+}$ and $\mathrm{Cu}^{2+}$ ions (added as $\mathrm{MgSO}_{4}$, $\mathrm{CaCl}_{2}, \mathrm{ZnSO}_{4}$ and $\mathrm{CuSO}_{4}$, respectively) in the final lipopeptide solutions was in the range of $0.2-2 \mathrm{mM}$. The surface tension of the lipopeptide solutions ( $\mathrm{pH} \sim 6.8-7.0)$ at the CMC $\left(\gamma_{\mathrm{cmc}}\right)$ was measured using a Krüss K20 Tensiometer (Krüss GmbH, Hamburg, Germany) at $25^{\circ} \mathrm{C}$, according to the du Noüy's ring method [29]. Each sample was measured in triplicates and averaged.

\subsection{Antimicrobial assays}

Antimicrobial activity was monitored by liquid growth inhibition as described previously [4]. The effect of metal ions on pseudofactin II activity was evaluated by liquid assay using Proteus mirabilis ATCC 22100 and Staphylococcus epidermidis KCTC 1917 incubated with pseudofactin II supplemented with divalent metal ions, namely $\mathrm{Ca}^{2+}(2.5 \mathrm{mM}), \mathrm{Mg}^{2+}(1 \mathrm{mM}), \mathrm{Cu}^{2+}(0.025 \mathrm{mM})$ and $\mathrm{Zn}^{2+}(0.02 \mathrm{mM})$. Luria-Bertani Broth (LB; $10 \mathrm{~g} \mathrm{~L}^{-1}$ bacto-tryptone, $5 \mathrm{~g} \mathrm{~L}^{-1}$ bacto-yeast extract, $10 \mathrm{~g} \mathrm{~L}^{-1} \mathrm{NaCl}$ ) was used for the antibacterial assay. Briefly, $50 \mu \mathrm{L}$ volumes of sterile double strength LB medium were dispensed into the wells of a 96-well microplate. Subsequently, $50 \mu \mathrm{L}$ of pseudofactin II $(0.036-0.48 \mathrm{mM})$ solution in phosphate-buffered saline (PBS) were added to the microplate wells and mixed with the medium. Bacteria incubated without pseudofactin II or incubated with metal ions addition were used as controls. All wells (except for negative controls) were inoculated with $2 \mu \mathrm{L}$ of overnight bacterial cultures (diluted to $\mathrm{OD}_{600}=0.1$ ) in LB medium, and the microplates were incubated for $24 \mathrm{~h}$ at $37^{\circ} \mathrm{C}$. After $24 \mathrm{~h}$ of incubation, the optical density at $600 \mathrm{~nm}$ of each well was measured using a Multiskan GO (Thermo Scientific) microplate spectrophotometer.

\subsection{Membrane permeabilization assay}

P. mirabilis and S. epidermidis harvested in the log phase were incubated in LB medium with pseudofactin II ( $0.24 \mathrm{mM})$ supplemented with the metal ions under study at normal physiological concentrations in eukaryotic cells: $\mathrm{Ca}^{2+} 2.5 \mathrm{mM} ; \mathrm{Mg}^{2+} 1 \mathrm{mM} ; \mathrm{Cu}^{2+}$ $0.025 \mathrm{mM}$; and $\mathrm{Zn}^{2+} 0.02 \mathrm{mM}$. Bacteria incubated without pseudofactin II or incubated with the divalent metal ions were used as controls. After $24 \mathrm{~h}$ at $37^{\circ} \mathrm{C}$, bacteria were washed with sterile PBS $(\mathrm{pH}=7.4)$. Then, the bacteria were stained for $30 \mathrm{~min}$ at $37^{\circ} \mathrm{C}$ with $1 \mathrm{~mL}$ of $0.6 \%$ reagents from the Live/Dead BacLight bacterial viability stain (L-7007, Invitrogen) prepared in PBS. Cells were visualized using an inverted Olympus IX53 fluorescence microscope (Japan). Experiments were repeated three times.

\section{Results and discussion}

\subsection{MS and UV-vis assignments}

An increasing number of reports have shown that peptides are able to create complexes with metal ions as calcium, copper, nickel, zinc and iron [30-32]. Taking into account the presence of amide bonds in the pseudofactin II structure, we evaluated its ability to bind metals forming metal-peptide complexes. For this purpose, pseudofactin II was incubated with $\mathrm{Mg}^{2+}, \mathrm{Ca}^{2+}, \mathrm{Zn}^{2+}$ or $\mathrm{Cu}^{2+}$ followed by analysis of the mixtures by MALDI-TOF mass
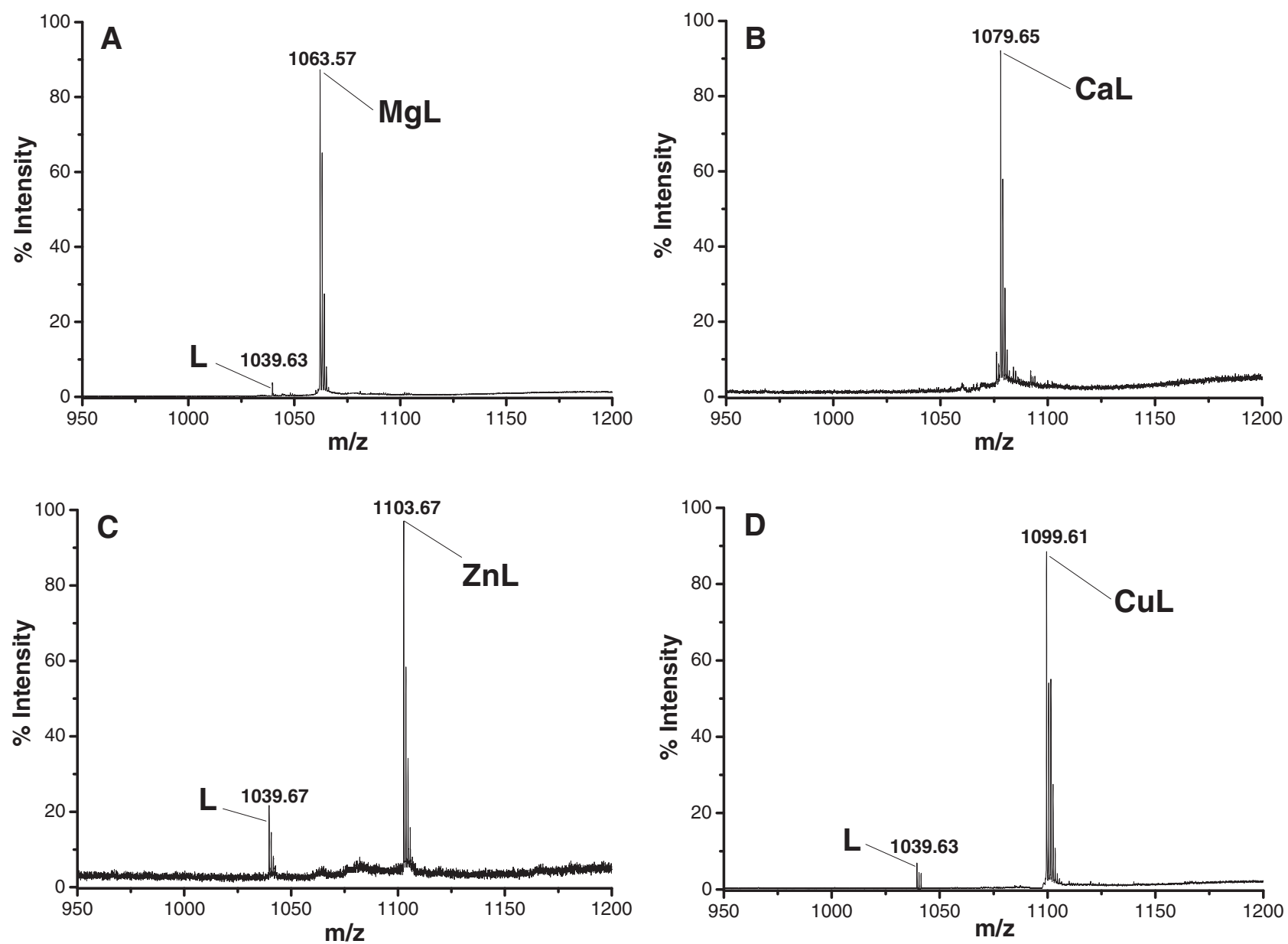

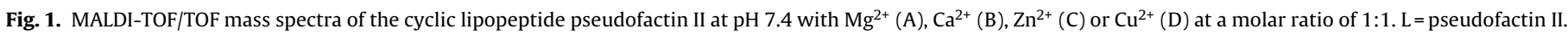


spectrometry (Fig. 1). The difference in molecular mass between pseudofactin II alone (1039.63 Da) and pseudofactin II in the presence of $\mathrm{Cu}^{2+}$ (1099.61 Da) suggest that divalent copper ions bind to the peptide at a molar ratio of 1:1 (Fig. 1D). Addition of an excess of this ion (molar ratio $\mathrm{n}_{\mathrm{L}}: \mathrm{n}_{\mathrm{Cu}}=1: 2$ ) did not promote the formation of di-nuclear complexes of pseudofactin II. The mass spectra showed that the additional equivalent of $\mathrm{Cu}^{2+}$ did not induce any discernible changes in the mass of the mononuclear complexes formed. A similar relationship between the molecular mass of the pseudofactin II and $\mathrm{Mg}^{2+}, \mathrm{Ca}^{2+}, \mathrm{Zn}^{2+}$ association was observed in Fig. $1 \mathrm{~A}-\mathrm{C}$. The absence of di-nuclear complexes further suggests the association of metal ions in the interior of the lipopeptide ring.

Likewise, Rautenbach and collaborators [33] reported the interaction of iturin $\mathrm{A}_{2}$ antimicrobial lipopeptide analogues with alkali metal ions as evaluated by electrospray ionization mass spectroscopy (ESI-MS). The authors showed that iturin $A_{2}$ and its shorter linear analogues linked with either one or two alkali metal cations, while the cyclic peptides linked with only one cation. Iturin $\mathrm{A}_{2}$ exhibits affinity for the alkali metal cations $\mathrm{Na}^{+}$and $\mathrm{K}^{+}$. However, this lipopeptide was found to precipitate in the presence of divalent cations as $\mathrm{Mg}^{2+}$ and $\mathrm{Ca}^{2+}$ [34].

At $\mathrm{pH} 6.5$ the d-d transition on the UV-vis spectrum of $\mathrm{Cu}^{2+}$-peptide complex was observed. Experimental $\lambda_{\max }=560 \mathrm{~nm}$ was very similar to the theoretical $\lambda_{\max }=561 \mathrm{~nm}$ calculated for $\left\{3 \mathrm{~N}^{-}, \mathrm{H}_{2} \mathrm{O}\right\}$ coordination mode in square planar complex [35] which is in agreement with the theoretical modelling study. With increasing $\mathrm{pH}$ no changes in spectroscopic parameters were observed (Table S1). Therefore, it can be concluded that the $\left\{3 \mathrm{~N}^{-}\right.$, $\mathrm{H}_{2} \mathrm{O}$ \} complex exists in the system for the whole basic range of $\mathrm{pH}$.

\subsection{CD spectroscopy}

As shown in Fig. 2, the addition of $\mathrm{Cu}^{2+}$ induces a slight change in the $C D$ curve until reaching a metal-ligand ratio of $1: 1$. Further additions of metal did not change the CD curve, which indicates that the reaction between $\mathrm{Cu}^{2+}$ and pseudofactin II is complete at one molar equivalent of $\mathrm{Cu}^{2+}$.

$\mathrm{CD}$ measurement was performed to monitor the secondary structure changes of pseudofactin II induced by the presence of divalent metal ions. The spectra were scanned over the wavelength range of $190-260 \mathrm{~nm}$ to probe transitions in the backbone amide. Fig. S1 presents the CD spectra of the lipopeptide with the divalent cations under study. These results indicate that the addition of $\mathrm{Mg}^{2+}$ and $\mathrm{Ca}^{2+}$ does not influence the structure of pseudofactin II. With the increase of $\mathrm{Zn}^{2+}$ and $\mathrm{Cu}^{2+}$ concentrations, the shape of the spectrum is changed, which could be interpreted as the occurrence of significant conformational modifications. Fitting the spectrum

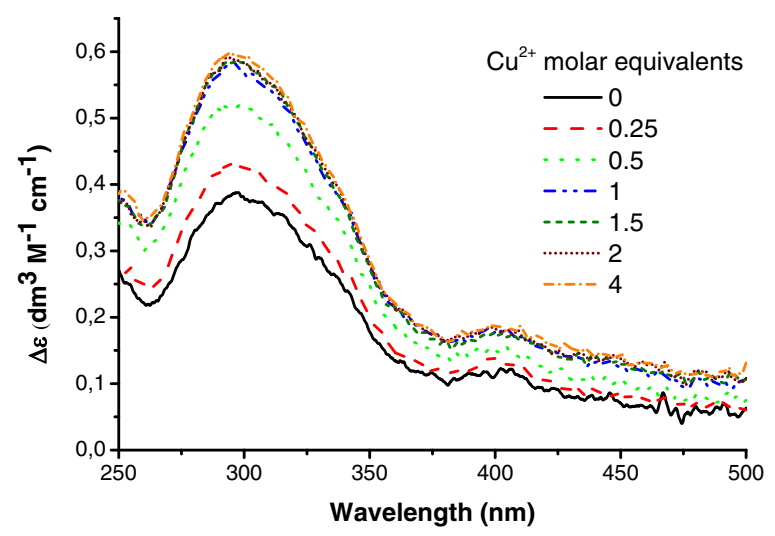

Fig. 2. Circular dichroism spectra. Titration of pseudofactin II with $\mathrm{Cu}^{2+}$ performed to determine at which $n_{L}: n_{C u}$ ratio the reaction between the peptide and the ion is complete. of free pseudofactin II at $25^{\circ} \mathrm{C}$ yielded a $36.75 \% \beta$-strand and only $0.43 \% \alpha$-helical structure. The addition of $4 \mathrm{mM}$ of $\mathrm{Cu}^{2+}$ and $8 \mathrm{mM}$ of $\mathrm{Zn}^{2+}$ to pseudofactin II caused a secondary structural change of the lipopeptide to $24.76 \% \beta$-strand $/ 1.5 \% \alpha$-helical and $22.01 \% \beta$ strand $/ 1.4 \% \alpha$-helical, respectively. These spectral changes suggest the occurrence of bulk conformational modifications due to metal ions-binding to the peptide backbone. Understanding the pseudofactin II conformational changes is of utmost relevance to develop improved analogues through the process of rational drug design.

\subsection{Molecular modelling simulation}

To analyze structural aspects of the binding properties of pseudofactin II to the metal ions under study, we performed semiempirical calculations. The possible conformations of the divalent metal ions-pseudofactin complexes were optimized at PM6 level of theory leading to several unique conformations. Herein we report only the most stable conformers. The theoretically optimal structure determined for the free pseudofactin II is presented in Fig. 3. As can be observed, the cycle of the lipopeptide evaluated might be partially stabilized by two hydrogen bonds, in which the $-\mathrm{O} \cdots \mathrm{H}-\mathrm{N}$ - distances are equal 2.17 and $1.84 \AA$, respectively.

Based on previous works concerning the effect of divalent cations on the structure of cyclic peptides and lipopeptides, we selected the most likely metal binding sites in the structure of the systems mentioned below. Fig. 3 contain the lowest-energy structures of metal ion-lipopeptide complexes established on the basis of semi-empirical calculations. It is worth of note that for all the considered complexes we are able to provide stable metal bonded conformers of the lipopeptide. In the case of $\mathrm{Cu}^{2+}$-pseudofactin complexes, we proposed two possible conformations. Our findings suggest that the structural parameters are highly dependent on the metal binding site. In both conformations presented below, copper is coordinated by three nitrogens, which is in good agreement with previous findings [16]. According to that study, amino and two amide groups of colistin were suggested to be involved in the copper ion binding [16]. The authors confirmed this hypothesis by spectroscopic measurements. Likewise, molecular modelling data also support our spectroscopic results for $\mathrm{Cu}^{2+}$ complexes with pseudofactin II. As shown in Fig. 3, both conformational structures exhibit the copper ions coordinated by three nitrogens in almost planar configurations and the difference between the total energies is about $1 \mathrm{kcal} \mathrm{mol}^{-1}$. It is also worth to mention that after ion metal binding mutual spatial arrangement of the hydrophobic and hydrophilic domains change. Similar results were obtained for the zinc-lipopeptide complexes. $\mathrm{Zn}^{2+}$ may be coordinated by four carbonyl oxygens in an almost tetrahedral configuration (Fig. 3).

Straus and collaborators suggested that calcium and magnesium cations can mask the negatively charged residues of lipopeptides, including the anionic daptomycin [36]. Based on previous works and molecular dynamic simulations, the authors concluded that Asp3, Asp7 and Asp9 residues are essential for the lipopeptide activity and also play an important role in $\mathrm{Ca}^{2+}$ binding, in which the calcium oxygen-distances were set to $2.5 \AA$. Interestingly, the substitution of $\mathrm{Mg}^{2+}$ ions does not influence any structural rearrangements in the lipopeptide molecule. According to our spectroscopic results, the addition of $\mathrm{Mg}^{2+}$ and $\mathrm{Ca}^{2+}$ does not influence the structure of pseudofactin II. The calculated structure in which the $\mathrm{Mg}^{2+}$ ion is coordinated by four oxygen donor atoms is presented in Fig. 3. In the case of $\mathrm{Ca}^{2+}$-pseudofactin II complexes, we were not able to obtain tetra- and tri-coordinated systems. The structure presented in Fig. 3 is characterized by two coordinated binding sites. Moreover, the structural differences upon metal binding in the case of these two metal complexes are smaller than for $\mathrm{Zn}^{2+}$ and $\mathrm{Cu}^{2+}$ structures obtained by molecular modelling simulations. 


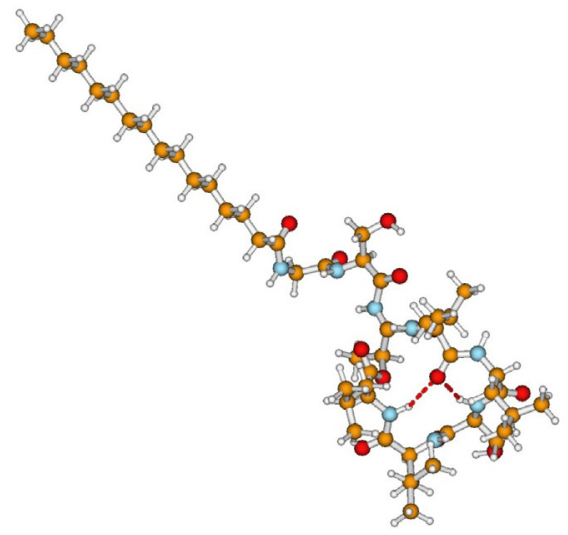

pseudofactin II

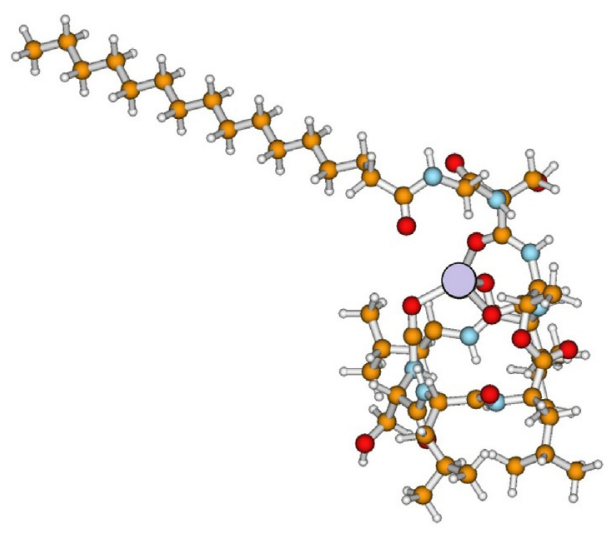

$\mathrm{Zn}^{2+}$-pseudofactin II

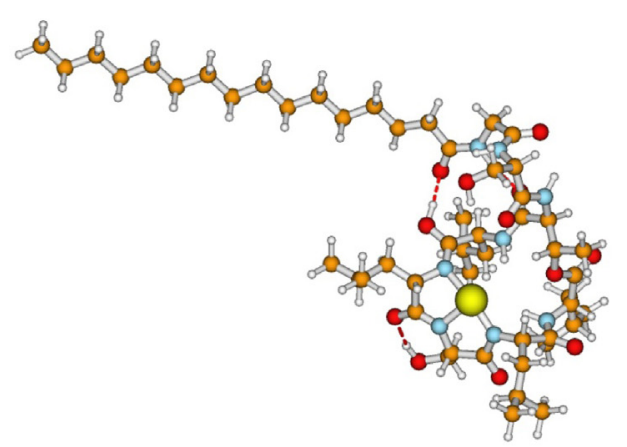

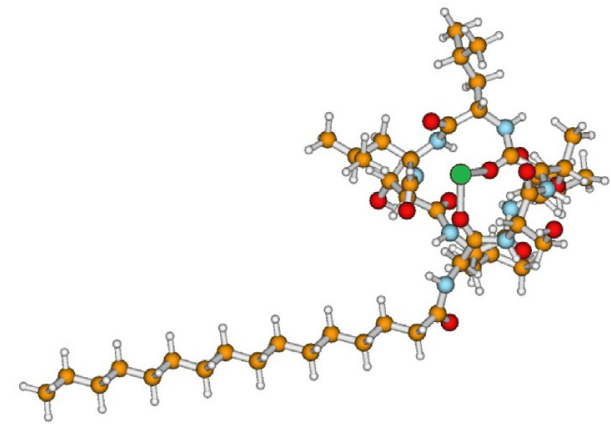

\section{$\mathrm{Ca}^{2+}$-pseudofactin II}

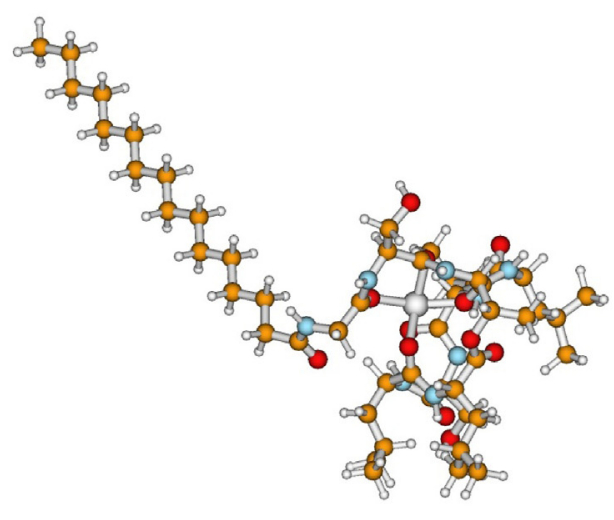

$\mathrm{Mg}^{2+}$-pseudofactin II

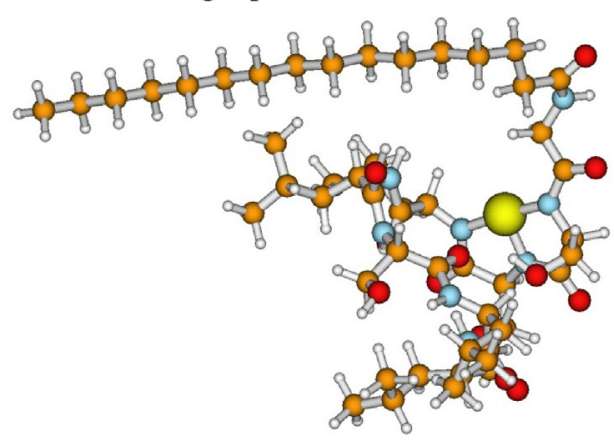

\section{$\mathrm{Cu}^{2+}$-pseudofactin II}

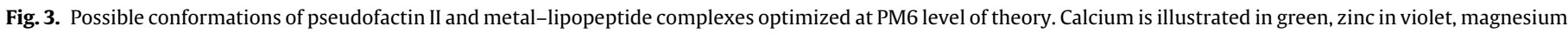

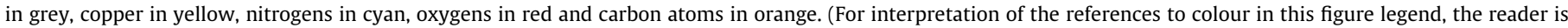
referred to the web version of this article.).

\subsection{Dynamic light scattering}

Our main interest in this evaluation was to understand the effect of divalent metal ions on the aggregation characteristics of pseudofactin II. Accordingly, DLS studies were performed for the lipopeptide solution at different divalent metal ions concentrations (Table 1 ). The concentration of pseudofactin II used in these studies $(0.3 \mathrm{mM})$ was selected taking into account the best PDI value (which indicates the width of the particle size distribution) obtained for different pseudofactin II concentrations (data not shown). The mean hydrodynamic diameter of pseudofactin II micelles $(0.3 \mathrm{mM})$ in water was $77.01 \mathrm{~nm}$, while PDI value was 0.195 . As shown in Table 1 , the micelle sizes remain almost constant with the variation of $\mathrm{Mg}^{2+}$ and $\mathrm{Ca}^{2+}$ concentrations. However, an increase of $\mathrm{Zn}^{2+}$ and $\mathrm{Cu}^{2+}$ concentrations from 0.15 to $8 \mathrm{mM}$ led to an increase of the micelles sizes from $83.11 \mathrm{~nm}$ to $263.6 \mathrm{~nm}$ and from $118.0 \mathrm{~nm}$ to $681.7 \mathrm{~nm}$, respectively. The bimodal size distribution at high $\mathrm{Zn}^{2+}$ and $\mathrm{Cu}^{2+}$ concentrations might have been caused by the transformation in structure of the micelles from spherical to cylindrical shape or to liposomes. This observation suggested 
Table 1

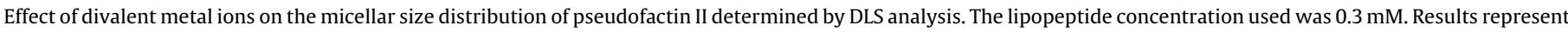
the mean of 9 replicates \pm SD.

\begin{tabular}{|c|c|c|c|c|c|c|c|c|}
\hline \multirow[t]{2}{*}{ Concentration(mM) } & \multicolumn{2}{|l|}{$\mathrm{Mg}^{2+}$} & \multicolumn{2}{|l|}{$\mathrm{Ca}^{2+}$} & \multicolumn{2}{|l|}{$\mathrm{Zn}^{2+}$} & \multicolumn{2}{|l|}{$\mathrm{Cu}^{2+}$} \\
\hline & Size (nm) & PDI & Size (nm) & PDI & Size (nm) & PDI & Size (nm) & PDI \\
\hline 0 & $77.0 \pm 2.2$ & $0.195 \pm 0.007$ & $77.0 \pm 2.2$ & $0.195 \pm 0.007$ & $77.0 \pm 2.2$ & $0.195 \pm 0.007$ & $77.0 \pm 2.2$ & $0.195 \pm 0.007$ \\
\hline 0.15 & $90.7 \pm 2.1$ & $0.121 \pm 0.010$ & $86.2 \pm 0.7$ & $0.303 \pm 0.021$ & $83.1 \pm 1.4$ & $0.248 \pm 0.007$ & $118.0 \pm 2.6$ & $0.167 \pm 0.009$ \\
\hline 0.3 & $94.2 \pm 1.1$ & $0.299 \pm 0.012$ & $116.8 \pm 0.7$ & $0.137 \pm 0.012$ & $138.6 \pm 2.3$ & $0.221 \pm 0.012$ & $134.2 \pm 2.7$ & $0.134 \pm 0.011$ \\
\hline 0.6 & $111.9 \pm 0.5$ & $0.184 \pm 0.009$ & $117.2 \pm 2.2$ & $0.175 \pm 0.004$ & $165.0 \pm 1.5$ & $0.078 \pm 0.009$ & $150.1 \pm 2.4$ & $0.172 \pm 0.005$ \\
\hline 1 & $112.1 \pm 0.2$ & $0.176 \pm 0.010$ & $118.0 \pm 1.5$ & $0.167 \pm 0.001$ & $185.8 \pm 1.8$ & $0.096 \pm 0.011$ & $212.4 \pm 0.5$ & $0.174 \pm 0.004$ \\
\hline 2 & $113.0 \pm 1.1$ & $0.187 \pm 0.012$ & $118.1 \pm 1.8$ & $0.191 \pm 0.022$ & $197.9 \pm 3.1$ & $0.098 \pm 0.006$ & $283.1 \pm 0.9$ & $0.194 \pm 0.003$ \\
\hline 4 & $114.7 \pm 1.0$ & $0.181 \pm 0.009$ & $119.7 \pm 2.5$ & $0.186 \pm 0.011$ & $207.4 \pm 2.6$ & $0.065 \pm 0.003$ & $368.0 \pm 2.1$ & $0.266 \pm 0.016$ \\
\hline 6 & $117.4 \pm 0.1$ & $0.196 \pm 0.014$ & $122.0 \pm 0.8$ & $0.160 \pm 0.013$ & $228.4 \pm 3.0$ & $0.086 \pm 0.001$ & $533.8 \pm 3.1$ & $0.266 \pm 0.011$ \\
\hline 8 & $119.4 \pm 0.6$ & $0.207 \pm 0.016$ & $122.9 \pm 1.3$ & $0.169 \pm 0.007$ & $263.6 \pm 1.8$ & $0.133 \pm 0.004$ & $681.7 \pm 2.8$ & $0.236 \pm 0.013$ \\
\hline
\end{tabular}

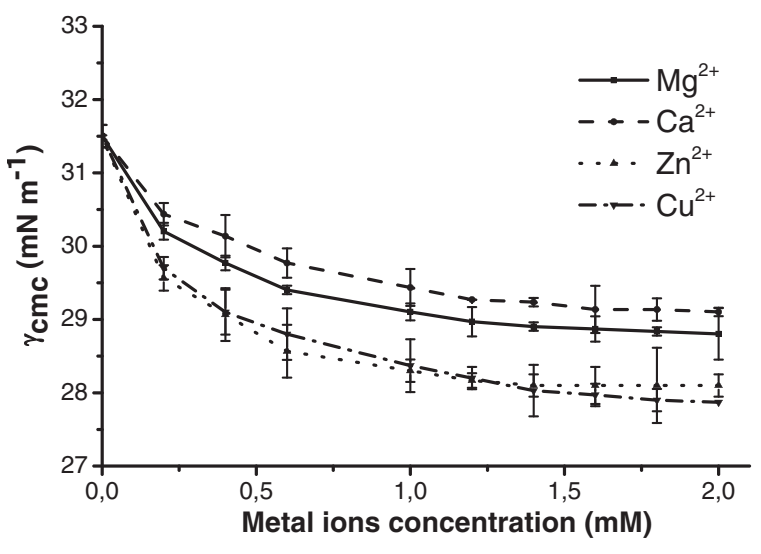

Fig. 4. Effect of metal ions on the surface tension at the CMC $\left(\gamma_{\mathrm{cmc}}\right)$ of pseudofactin II solution (lipopeptide concentration was at its critical micelle concentration, $\mathrm{CMC}=0.07 \mathrm{mM}$ ). The values represent the mean of triplicates $\pm \mathrm{SD}$.

that the addition of divalent transition metal ions helped smaller micelles to form larger micellar aggregates. Probably, the interaction between $\mathrm{Zn}^{2+}$ and $\mathrm{Cu}^{2+}$ lead to a greater screening of charge on polar head groups and less electrostatic repulsion between polar head groups of lipopeptides in the micelle.

\subsection{Surface tension}

In order to evaluate the effect of different concentrations of the divalent metal ions $\mathrm{Ca}^{2+}, \mathrm{Mg}^{2+}, \mathrm{Zn}^{2+}$ and $\mathrm{Cu}^{2+}$ on the surface activity of the lipopeptide pseudofactin II, preliminary studies were performed using a du Noüy's ring tensiometer. In our experiment, different amount of metal ions were added to the lipopeptide solution containing $0.07 \mathrm{mM}$ of pseudofactin II (the CMC of this biosurfactant). Fig. 4 shows the relation between the surface tension $\left(\gamma_{\mathrm{cmc}}\right)$ values of pseudofactin II and the respective metal complexes at $25^{\circ} \mathrm{C}$.

The surface tension values at the CMC $\left(\gamma_{\mathrm{cmc}}\right)$ of pseudofactin II complexes were found to be lower than the obtained for the free lipopeptide. Increasing the concentration of $\mathrm{Cu}^{2+}$ led to a significant reduction in the $\gamma_{\mathrm{cmc}}$ of pseudofactin II; similar reductions in the $\gamma_{\mathrm{cmc}}$ with increasing concentrations of $\mathrm{Zn}^{2+}, \mathrm{Ca}^{2+}$ and $\mathrm{Mg}^{2+}$ were also observed (Fig. 4). These results suggest that divalent cations can greatly enhance the close packing of the pseudofactin II molecules at the air-water interface, and are similar to those reported for the surface tension changes of surfactin and iturin with $\mathrm{Ca}^{2+}$ ions [37]. Furthermore, Arutchelvi and co-workers observed that the CMC of surfactin was reduced by the addition of $\mathrm{Ni}^{2+}, \mathrm{Zn}^{2+}$, $\mathrm{Cd}^{2+}$ or $\mathrm{Ca}^{2+}$ due to the overcome of the electrostatic repulsive force between the ionic surfactin micelles [38].

The stability of micelles is governed by a balance of electrostatic and hydrophobic interactions. The hydrophobic effect is responsible for the removal of fatty acid chain from the aqueous environment and favors the micellization [38]. This fact could be explained from the unique properties of the metal complexes in water, i.e. the complexes retain its unity in their solutions, which increased their volume in the aqueous media leading to repulsion between the hydrophobic chains and the water molecules. In summary, these studies revealed that all the tested metal ions led to reductions of the surface tension at the $\mathrm{CMC}\left(\gamma_{\mathrm{cmc}}\right)$ values of pseudofactin II, being the highest reductions obtained with $\mathrm{Cu}^{2+}$ and $\mathrm{Zn}^{2+}$ (from $31.5 \mathrm{mN} \mathrm{m}^{-1}$ up to around $28 \mathrm{mN} \mathrm{m}^{-1}$ ).

\subsection{Effect of divalent metal ions on the antimicrobial pseudofactin II activity}

Pseudofactin II antimicrobial activity was tested in the presence or absence of metal ions. The results demonstrated that the
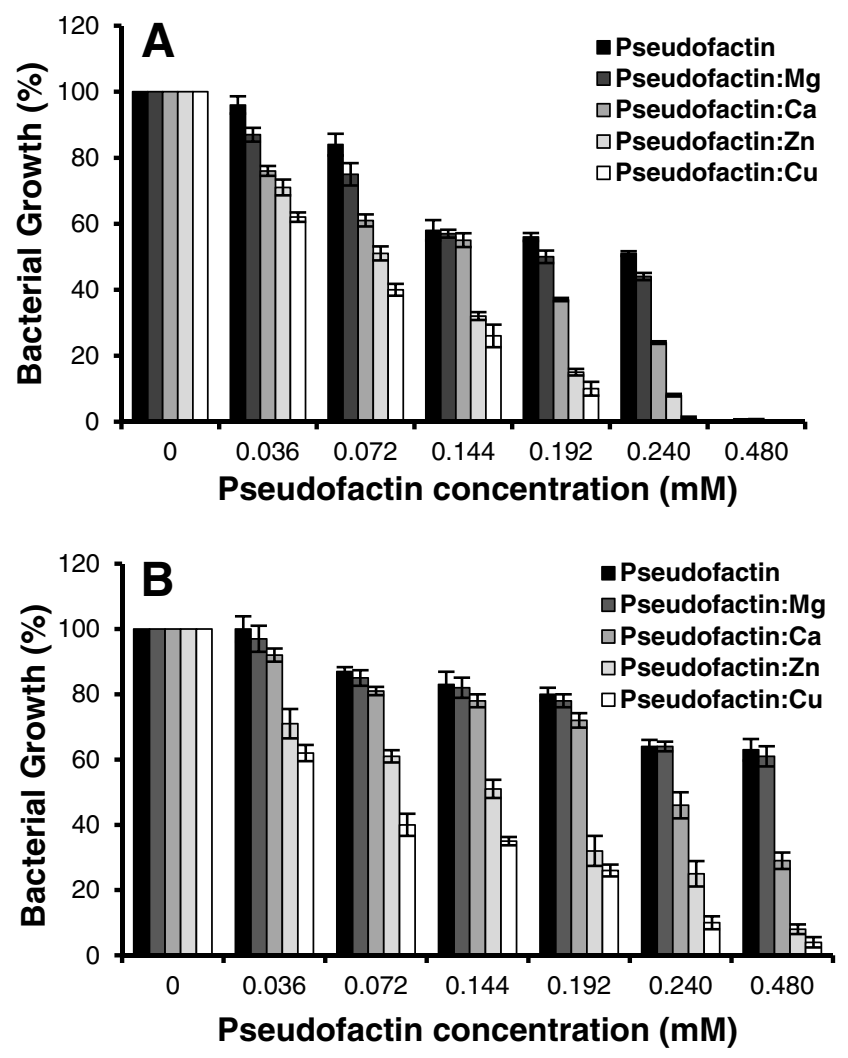

Fig. 5. Effect of $\mathrm{Mg}^{2+}, \mathrm{Ca}^{2+}, \mathrm{Zn}^{2+}$ and $\mathrm{Cu}^{2+}$ supplementation on the growth of Staphylococcus epidermidis (A) and Proteus mirabilis (B). Bacteria were incubated in serial dilutions of pseudofactin II $(0.036-0.48 \mathrm{mM})$ without or supplemented with $1 \mathrm{mM}$ $\mathrm{MgSO}_{4}, 2.5 \mathrm{mM} \mathrm{CaCl}_{2}, 0.02 \mathrm{mM} \mathrm{ZnSO}_{4}$ and $0.025 \mathrm{mM} \mathrm{CuSO}_{4}$ for $24 \mathrm{~h}$ at $37{ }^{\circ} \mathrm{C}$. 
metal ions herein studied, when added in normal human physiological concentrations to the culture media, increase the activity of pseudofactin II against microorganisms with multidrug-resistant profiles, especially S. epidermidis (Fig. 5A) and P. mirabilis (Fig. 5B). The interaction of the lipopeptide with the lipid bilayer of bacterial membranes may be facilitated by metal ions, both by neutraliz-

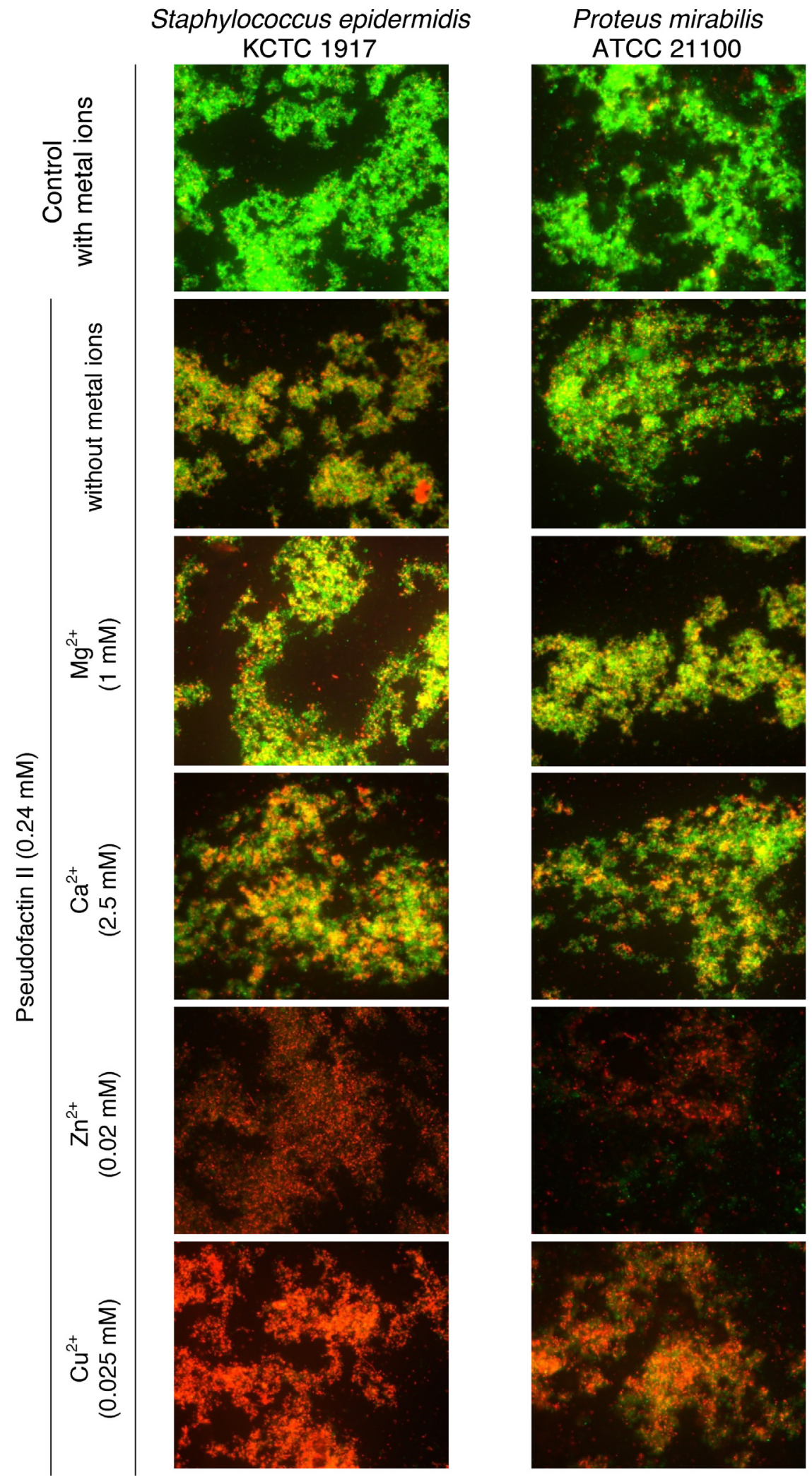

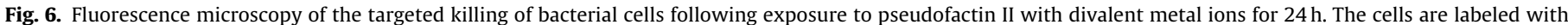

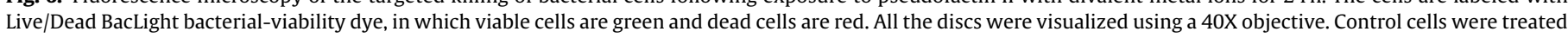
with mixtures of metal ions. (For interpretation of the references to colour in this figure legend, the reader is referred to the web version of this article.) 
ing the anionic charges and by favoring its association with the membrane head groups [39]. The results suggest the possible use of this cyclic lipopeptide as a potential antimicrobial agent in medical applications against microorganisms responsible for diseases and infections, thus making it a suitable alternative to conventional antibiotics.

\subsection{Pseudofactin II increases cell membrane permeability}

Because several lipopeptide biosurfactants exert their effect by membrane permeabilization [40-42], we investigated whether the membrane might be the target of pseudofactin II. The permeability of the cytoplasmic membrane was visualized after cells staining with two fluorescent nucleic acid stains, SYTO-9 and propidium iodide (PI). Viable cells with intact cytoplasmic membrane appear green when the membrane-permeable stain SYTO-9 enters the cells. Bacterial cells with compromised membranes are stained red by propidium iodide, a membrane-impairment stain. The untreated $S$. epidermidis and $P$. mirabilis cells were stained green, whereas the few bacterial cells treated by pseudofactin II at $0.24 \mathrm{mM}$ for $24 \mathrm{~h}$ became red, as shown in Fig. 6. However, bacteria treated with pseudofactin II supplemented with divalent metal cations were damaged. These results clearly show increasing permeability of the cytoplasmic membrane after Gram-positive and Gram-negative cells were treated with the ion metal-lipopeptide complexes (Fig. 6).

In our opinion, the significant higher antimicrobial activity of the pseudofactin II metal complexes is attributed to disruption of the cytoplasmic membrane of both Gram-negative and Gram-positive bacteria. Probably, the binding of divalent ions causes deeper penetration into the membrane and aggregation of pseudofactin II creating channels that allow the ions to flow into and out of the cells. This leads to membrane depolarization, which results in disruption of cellular activity and rapid cell death. Despite the evidence of cell membrane damage, this study does not exclude additional bactericidal mechanisms potentially exerted by pseudofactin II.

\section{Conclusions}

To the best of our knowledge, this is the first report on pseudofactin II as the ligand design for selective complexation of metal ions. In this work, we have investigated the interaction between series of doubly charged metal cations and pseudofactin II by means of mass spectroscopy, UV-vis and CD spectra, molecular modelling, DLS and surface tension. For all the tested divalent metal ions, only mononuclear complexes were obtained even when the metal ions were used at a molar ratio of $1: 2$. The $C D$ data showed the partial loss of the secondary structure of pseudofactin II upon $\mathrm{Zn}^{2+}$ and $\mathrm{Cu}^{2+}$ addition. In addition, molecular modelling analysis confirmed that the structural parameters are highly dependent on the metal binding site. From the DLS results, it could be concluded that the addition of divalent transition metal ions helped smaller micelles to form larger micellar aggregates according to the following order $\mathrm{Cu}^{2+}$-pseudofactin $>\mathrm{Zn}^{2+}$-pseudofactin $>\mathrm{Ca}^{2+}$-pseudofactin $>$ $\mathrm{Mg}^{2+}$-pseudofactin. Moreover, the pseudofactin II metal complexes exhibited lower surface tension values as compared to the pseudofactin II itself. Finally, the specific binding of pseudofactin II to copper and zinc ions greatly increased the antimicrobial activity against uropathogenic bacteria comparing to the lipopeptide alone.

In conclusion, the interaction between divalent metal cations and pseudofactin II herein studied clearly explains the pharmacological activity of the lipopeptide, especially its antibacterial properties. We believe that our results may also be relevant to understand the metal-bound active biological form of pseudofactin II.

\section{Declaration of interest}

The authors report no conflicts of interest. The authors are responsible for the content and writing of the manuscript.

\section{Acknowledgments}

Author Żaneta Czyżnikowska gratefully acknowledge the allotment of the CPU time in Wroclaw Center of Networking and Supercomputing (WCSS). Lígia Rodrigues and Eduardo Gudiña acknowledge the Fundação para a Ciência e Tecnologia (FCT) for the strategic funding of UID/BIO/04469/2013 unit and COMPETE 2020 (POCI-01-0145-FEDER-006684).

\section{Appendix A. Supplementary data}

Supplementary data associated with this article can be found, in the online version, at http://dx.doi.org/10.1016/j.colsurfb.2016.06. 055.

\section{References}

[1] J.M. Raaijmakers, I. De Bruijn, O. Nybroe, M. Ongena, Natural functions of lipopeptides from Bacillus and Pseudomonas: more than surfactants and antibiotics, FEMS Microbiol. Rev. 34 (2010) 1037-1062.

[2] P. Biniarz, M. Lukaszewicz, T. Janek, Screening concepts, characterization and structural analysis of microbial-derived bioactive lipopeptides: a review, Crit. Rev. Biotechnol. (2016), http://dx.doi.org/10.3109/07388551.2016.1163324.

[3] T. Janek, M. Lukaszewicz, T. Rezanka, A. Krasowska, Isolation and characterization of two new lipopeptide biosurfactants produced by Pseudomonas fluorescens BD5 isolated from water from the Arctic Archipelago of Svalbard, Bioresour. Technol. 101 (2010) 6118-6123.

[4] T. Janek, M. Lukaszewicz, A. Krasowska, Antiadhesive activity of the biosurfactant pseudofactin II secreted by the Arctic bacterium Pseudomonas fluorescens BD5, BMC Microbiol. 12 (2012) 24.

[5] T. Janek, A. Krasowska, A. Radwanska, M. Lukaszewicz, Lipopeptide biosurfactant pseudofactin II induced apoptosis of melanoma A 375 cells by specific interaction with the plasma membrane, PLoS One 8 (2013) e57991.

[6] M.S. Glover, J.M. Dilger, F. Zhu, D.E. Clemmer, The binding of $\mathrm{Ca}^{2+}, \mathrm{Co}^{2+}, \mathrm{Ni}^{2+}$ $\mathrm{Cu}^{2+}$, and $\mathrm{Zn}^{2+}$ cations to angiotensin I determined by mass spectrometry based techniques, Int. J. Mass Spectrom. 354 (2013) 318-325.

[7] T.D. Son, M. Roux, M. Ellenberger, Interaction of $\mathrm{Mg}^{2+}$ ions with nucleoside triphosphates by phosphorus magnetic resonance spectroscopy, Nucleic Acids Res. 2 (1975) 1101-1110.

[8] A.H. Wong, D. Zhou, J.M. Rini, The X-ray crystal structure of human aminopeptidase $\mathrm{N}$ reveals a novel dimer and the basis for peptide processing, J. Biol. Chem. 287 (2012) 36804-36813.

[9] A. Okado-Matsumoto, I. Fridovich, Subcellular distribution of superoxide dismutases (SOD) in rat liver: Cu, Zn-SOD in mitochondria, J. Biol. Chem. 276 (2001) 38388-38393.

[10] J. Parkash, K. Asotra, Calcium wave signaling in cancer cells, Life Sci. 87 (2010) 587-595.

[11] N. Raman, V. Muthuraj, S. Ravichandran, A. Kulandaisamy, Synthesis, characterisation and electrochemical behaviour of $\mathrm{Cu}(\mathrm{II}) \mathrm{Co}(\mathrm{II}), \mathrm{Ni}(\mathrm{II})$ and $\mathrm{Zn}$ (II) complexes derived from acetylacetone andp-anisidine and their antimicrobial activity, J. Chem. Sci. 115 (2003) 161-167.

[12] Z.H. Chohan, M. Arif, M.A. Akhtar, C.T. Supuran, Metal-based antibacterial and antifungal agents: synthesis, characterization, and in vitro biological evaluation of $\mathrm{Co}(\mathrm{II}), \mathrm{Cu}(\mathrm{II}), \mathrm{Ni}(\mathrm{II})$, and $\mathrm{Zn}(\mathrm{II})$ complexes with amino acid-derived compounds, Bioinorg. Chem. Appl. 2006 (2006) 13.

[13] A. Prakash, D. Adhikari, Application of Schiff bases and their metal complexes-a review, Int. J. Chem. Tech. Res. 3 (2011) 1891-1896.

[14] M.E. Falagas, S.K. Kasiakou, Colistin: the revival of polymyxins for the management of multidrug-resistant gram-negative bacterial infections, Clin. Infect. Dis. 40 (2005) 1333-1341.

[15] A.K. Dhariwal, M.S. Tullu, Colistin: re-emergence of the 'forgotten' antimicrobial agent, J. Postgrad. Med. 59 (2013) 208-215.

[16] K. Stokowa-Soltys, A. Kasprowicz, J. Wrzesinski, J. Ciesiolka, N. Gaggelli, E. Gaggelli, G. Valensin, M. Jezowska-Bojczuk, Impact of Cu ions on the structure of colistin and cell-free system nucleic acid degradation, J. Inorg. Biochem. 151 (2015) 67-74.

[17] G. Laverty, S.P. Gorman, B.F. Gilmore, The potential of antimicrobial peptides as biocides, Int. J. Mol. Sci. 12 (2011) 6566-6596. 
[18] T. Kelesidis, R. Humphries, D.Z. Uslan, D.A. Pegues, Daptomycin nonsusceptible enterococci: an emerging challenge for clinicians, Clin. Infect. Dis. 52 (2011) 228-234

[19] C. Vilhena, A. Bettencourt, Daptomycin: a review of properties, clinical use, drug delivery and resistance, Mini Rev. Med. Chem. 12 (2012) 202-209.

[20] E. Topchiy, T. Lehmann, Chelation of $\mathrm{Ca}^{2+}$ ions by a peptide from the repeat region of the Plasmodium falciparum circumsporozoite protein, Malar. J. 13 (2014) 195.

[21] E. Vass, F. Besson, Z. Majer, L. Volpon, M. Hollosi, Ca ${ }^{2+}$-induced changes of surfactin conformation: a FTIR and circular dichroism study, Biochem. Biophys. Res. Commun. 282 (2001) 361-367.

[22] G. Bunkoczi, L. Vertesy, G.M. Sheldrick, Structure of the lipopeptide antibiotic tsushimycin, Acta Crystallogr. D Biol. Crystallogr. 61 (2005) 1160-1164.

[23] C. Louis-Jeune, M.A. Andrade-Navarro, C. Perez-Iratxeta, Prediction of protein secondary structure from circular dichroism using theoretically derived spectra, Proteins 80 (2011) 374-381.

[24] J.J.P. Stewart, Application of the PM6 method to modeling proteins, J. Mol. Model. 15 (2009) 765-805.

[25] E. Cances, B. Mennucci, J. Tomasi, A new integral equation formalism for the polarizable continuum model: theoretical background and applications to isotropic and anisotropic dielectrics, J. Chem. Phys. 107 (1997) 3032-3041.

[26] J. Tomasi, B. Mennucci, R. Cammi, Quantum mechanical continuum solvation models, Chem. Rev. 105 (2005) 2999-3094.

[27] J. Tomasi, B. Mennucci, E. Cances, The IEF version of the PCM solvation method: an overview of a new method addressed to study molecular solutes at the QM ab initio level, J. Mol. Struct. Theochem 464 (1999) 211-226.

[28] M. Frisch, G.W. Trucks, H.B. Schlegel, G.E. Scuseria, M.A. Robb, J.R. Cheeseman, G. Scalmani, V. Barone, B. Mennucci, G.A. Petersson, Gaussian 09, Revision A. 02, 270, Gaussian Inc., Wallingford CT, 2009, pp. 271.

[29] C. Huh, S.G. Mason, A rigorous theory of ring tensiometry, Colloid. Polym. Sci. 253 (1975) 566-580.

[30] D. Brewer, G. Lajoie, Evaluation of the metal binding properties of the histidine-rich antimicrobial peptides histatin 3 and 5 by electrospray ionization mass spectrometry, Rapid Commun. Mass Spectrom. 14 (2000) 1736-1745.

[31] M. Nishikawa, K. Ogawa, Antimicrobial activity of a chelatable poly(arginyl-histidine) produced by the ergot fungus Verticillium kibiense, Antimicrob. Agents Chemother. 48 (2004) 229-235.
[32] S. Farnaud, C. Rapisarda, T. Bui, A. Drake, R. Cammack, R.W. Evans, Identification of an iron-hepcidin complex, Biochem. J. 413 (2008) 553-557.

[33] M. Rautenbach, P. Swart, M.J. van der Merwe, The interaction of analogues of the antimicrobial lipopeptide iturin A2, with alkali metal ions, Bioorg. Med. Chem. 8 (2000) 2539-2548.

[34] F. Besson, G. Michel, Influence of divalent ions on the solubility of iturin and bacillomycin L, antifungal peptidolipids of Bacillus subtilis, Microbios 65 (1991) 15-21.

[35] E. Prenesti, P.G. Daniele, M. Prencipe, G. Ostacoli, Spectrum-structure correlation for visible absorption spectra of copper (II) complexes in aqueous solution, Polyhedron 18 (1999) 3233-3241.

[36] S.W. Ho, D. Jung, J.R. Calhoun, J.D. Lear, M. Okon, W.R. Scott, R.E. Hancock, S.K. Straus, Effect of divalent cations on the structure of the antibiotic daptomycin, Eur. Biophys. J. 37 (2008) 421-433.

[37] J. Arutchelvi, J. Sangeetha, J. Philip, M. Doble, Self-assembly of surfactin in aqueous solution: role of divalent counterions, Colloids Surf. B Biointerfaces 116 (2014) 396-402.

[38] V. Rangarajan, G. Dhanarajan, R. Sen, Improved performance of cross-flow ultrafiltration for the recovery and purification of $\mathrm{Ca}^{2+}$ conditioned lipopeptides in diafiltration mode of operation, J. Membr. Sci. 454 (2014) 436-443.

[39] K.S. Rotondi, L.M. Gierasch, A well-defined amphipathic conformation for the calcium-free cyclic lipopeptide antibiotic daptomycin, in aqueous solution, Biopolymers 80 (2005) 374-385.

[40] C. Carrillo, J.A. Teruel, F.J. Aranda, A. Ortiz, Molecular mechanism of membrane permeabilization by the peptide antibiotic surfactin, Biochim. Biophys. Acta 1611 (2003) 91-97.

[41] F.J. Aranda, J.A. Teruel, A. Ortiz, Further aspects on the hemolytic activity of the antibiotic lipopeptide iturin A, Biochim. Biophys. Acta 1713 (2005) 51-56.

[42] A. Clausell, M. Garcia-Subirats, M. Pujol, M.A. Busquets, F. Rabanal, Y. Cajal, Gram-negative outer and inner membrane models: insertion of cyclic cationic lipopeptides, J. Phys. Chem. B 111 (2007) 551-563. 